J. Nonlinear Var. Anal. 4 (2020), No. 3, pp. 337-355

Available online at http://jnva.biemdas.com

https://doi.org/10.23952/jnva.4.2020.3.02

\title{
STRONG CONVERGENCE OF INERTIAL MANN ALGORITHMS FOR SOLVING HIERARCHICAL FIXED POINT PROBLEMS
}

\author{
BING TAN*, SONGXIAO LI \\ Institute of Fundamental and Frontier Sciences, \\ University of Electronic Science and Technology of China, Chengdu, China
}

\begin{abstract}
The paper introduces two inertial Mann algorithms to find solutions of hierarchical fixed point problems of nonexpansive mappings. We obtain strong convergence theorems in Hilbert spaces under suitable conditions. Some numerical examples are provided to illustrate the numerical behavior of the algorithms and numerical results show that our proposed algorithms are efficient and robust.

Keywords. Hierarchical fixed point problem; Inertial Mann algorithm; Nonexpansive mapping; Strong convergence; Viscosity method.
\end{abstract}

\section{INTRODUCTION}

Throughout this paper, $C$ is assumed to be a nonempty convex and closed set in a real Hilbert space $H$. The inner product and the corresponding induced norm are represented by $\langle\cdot, \cdot\rangle$ and $\|\cdot\|$, respectively. Let $I$ be the identity mapping on $C$ and let $P_{C}$ be the metric projection of $H$ onto $C$. To begin with, let us recall the following concepts in convex and nonlinear analysis. For all $x, y \in C$, a mapping $A: C \rightarrow H$ is said to be (i) monotone iff $\langle A x-A y, x-y\rangle \geq 0$; (ii) strongly monotone iff $\langle A x-A y, x-y\rangle \geq k\|x-y\|^{2}$, where $k$ is a real constant; (iii) Lipschitzian if $\|A x-A y\| \leq L\|x-y\|$, where $L>0$ is some real number. Let us also recall that a mapping $T: C \rightarrow C$ is said to be (i) contraction if $\|T x-T y\| \leq \rho\|x-y\|$, where $\rho$ is a real number in $(0,1)$; (ii) nonexpansive if $\|T x-T y\| \leq\|x-y\|$. The set of fixed points of $T$ is denoted by $\operatorname{Fix}(T)$, that is, $\operatorname{Fix}(T):=\{x \in C: T x=x\}$.

In 2006, Moudafi and Mainge [11] introduced the following hierarchical fixed point problem (in short, HFPP)

$$
\text { find } x^{*} \in \operatorname{Fix}(T) \quad \text { such that }\left\langle x^{*}-x,(I-S) x^{*}\right\rangle \leq 0, \quad \forall x \in \operatorname{Fix}(T)
$$

for a nonexpansive mapping $T$ with respect to another nonexpansive mapping $S$ on $C$. Let $\Omega:=\left\{x^{*} \in C:\left(P_{\operatorname{Fix}(T)} \circ S\right) x^{*}=x^{*}\right\}$ denote the solution set of (HFPP). It is not hard to check that solving (HFPP) is equivalent to the fixed point problem:

$$
\text { find } x^{*} \in C \quad \text { such that } x^{*}=\left(P_{\operatorname{Fix}(T)} \circ S\right) x^{*} \text {. }
$$

\footnotetext{
*Corresponding author.
}

E-mail addresses: bingtan72@gmail.com (B. Tan), jyulsx@163.com (S. Li).

Received November 20, 2019; Accepted October 18, 2020.

(C)2020 Journal of Nonlinear and Variational Analysis 
In addition, we can prove that (HFPP) is equivalent to the variational inclusion problem:

$$
\text { find } x^{*} \in C \quad \text { such that } 0 \in N_{\operatorname{Fix}(T)} x^{*}+(I-S) x^{*} \text {, }
$$

where $N_{\operatorname{Fix}(T)}$ is the normal cone of $\operatorname{Fix}(T)$, which is defined by

$$
N_{\operatorname{Fix}(T)}= \begin{cases}\{u \in H:\langle u, y-x\rangle \leq 0, \forall y \in \operatorname{Fix}(T)\}, & \text { if } x \in \operatorname{Fix}(T), \\ \emptyset, & \text { otherwise. }\end{cases}
$$

Problem (HFPP) covers hierarchical minimization problems, monotone variational inequality on fixed point sets, minimization problems over equilibrium constraints. Recently, some authors have studied the existence of hierarchical fixed points and their iterative algorithms; see, e.g., $[5,11,12,13,20,26]$ and the references therein. By setting $S=I-\gamma A$, where mapping $A$ is $\eta$-Lipschitzian and $k$-strongly monotone with $\gamma \in\left(0,2 k / \eta^{2}\right)$, one finds that (HFPP) is reduced to the so-called hierarchical variational inequality problem:

$$
\text { find } x^{*} \in \operatorname{Fix}(T) \quad \text { such that }\left\langle A\left(x^{*}\right), x-x^{*}\right\rangle \geq 0, \quad \forall x \in \operatorname{Fix}(T) .
$$

In order to solve (HFPP), Moudafi [12] introduced the following Mann iterative algorithm

$$
x_{n+1}=\left(1-\psi_{n}\right) x_{n}+\psi_{n}\left(\left(1-v_{n}\right) T x_{n}+v_{n} S x_{n}\right), \quad \forall n \geq 0,
$$

where $\left\{v_{n}\right\}$ and $\left\{\psi_{n}\right\}$ are two sequences in $(0,1)$. It is worth mentioning that some algorithms in signal processing and image reconstruction can be written as the Mann iteration and that the main feature of its corresponding convergence theorems provides a unified frame for analyzing various concrete algorithms; see, e.g., [6, 15, 16, 22, 23, 27].

For solving problem (HFPP), using the iterative algorithm (1.1), Moudafi [12] proved a weak convergence theorem. In particular, when mapping $S$ is a contraction, a special case of nonexpansive mappings, Yao and Liou obtained a strong convergence result [25]. Recently, Mainge and Moudafi [13] introduced the following iterative algorithm (1.2):

$$
\begin{aligned}
& \text { Algorithm } 1 \text { (Viscosity iterative algorithm (VIA)). } \\
& \quad x_{n+1}=\psi_{n} f\left(x_{n}\right)+\left(1-\psi_{n}\right)\left(v_{n} S x_{n}+\left(1-v_{n}\right) T x_{n}\right), \quad \forall n \geq 0, \quad \text { (1.2) }
\end{aligned}
$$

where $\left\{v_{n}\right\}$ and $\left\{\psi_{n}\right\}$ are two sequences in $(0,1)$, and mapping $f: C \rightarrow C$ is a contraction. They proved that under appropriate conditions the iterative sequence $\left\{x_{n}\right\}$ generated by (1.2) converges strongly to $x^{*} \in \operatorname{Fix}(T)$ which solves (HFPP). Motivated and inspired by [13], Yao, Liou and Marino [26] introduced a two-step iterative approach to solve the (HFPP):

$$
\begin{aligned}
& \text { Algorithm } 2 \text { (Two-step iterative algorithm (TWIA)). } \\
& \qquad \begin{array}{c}
y_{n}=v_{n} S x_{n}+\left(1-v_{n}\right) x_{n}, \\
x_{n+1}=\psi_{n} f\left(x_{n}\right)+\left(1-\psi_{n}\right) T y_{n}, \quad \forall n \geq 0
\end{array}
\end{aligned}
$$

They proved that under appropriate assumptions iterative algorithm (1.3) converges strongly to some fixed point of $T$, which solves (HFPP). It is worth noting that (1.3) is different from (1.2).

In view of the applications to the signal processing and the image reconstruction, it is important and interesting to construct some new iterative algorithms for solving (HFPP). In general, the convergence rate of Mann algorithm is slow. The fast convergence of algorithms is required in many practical applications. In particular, an inertial type extrapolation was first proposed by Polyak [14] as an acceleration process. In recent years, some authors constructed various fast 
iterative algorithms with the aid of inertial extrapolation techniques, such as, inertial Mann algorithms [19], inertial forward-backward splitting algorithms [17], inertial extragradient algorithms [9], inertial gradient algorithms [18], inertial projection algorithms [10, 21] and fast iterative shrinkage-thresholding algorithm (FISTA) [3].

Inspired and motivated by the above results, we introduce two inertial iterative algorithms for solving (HFPP). Strong convergence theorems are established in the framework of real Hilbert spaces. We also give some numerical examples to illustrate the computational performance of our proposed algorithms over some previously known algorithms in [13, 26]. Our results improves the corresponding results of Mainge and Moudafi [13], Yao et al. [26], Dong et al. [7].

This paper is organized as follows. Section 2 gives the mathematical preliminaries. Section 3 and Section 4 present two algorithms for solving (HFPP) and analyze their convergence. Section 5 numerically compares the behaviors of the proposed algorithms and the existing ones. Section 6, the last section, concludes the paper with a brief summary.

\section{PRELIMinaries}

Throughout this paper, we denote the strong and weak convergence of a sequence $\left\{x_{n}\right\}$ to a point $x \in H$ by $x_{n} \rightarrow x$ and $x_{n} \rightarrow x$, respectively. A set-valued operator $F: H \rightarrow 2^{H}$ is said to be (i) monotone if $\langle m-n, x-y\rangle \geq 0, \forall m \in F(x), n \in F(y)$; (ii) maximal monotone if the $\operatorname{graph} F:=\{(x, y) \in H \times H: y \in F(x)\}$ is not properly contained in the graph of any other monotone operator. An operator sequence $\left\{F_{n}\right\}$ is said to be graph convergent to $F$ if $\left\{\right.$ graph $\left.\left\{F_{n}\right\}\right\}$ converges to $\operatorname{graph}\{F\}$ in the Kuratowski-Painleve's sense, i.e., $\limsup _{n} \operatorname{graph}\left\{F_{n}\right\} \subset \operatorname{graph}\{F\} \subset \liminf _{n} \operatorname{graph}\left\{F_{n}\right\}$.

The following lemmas will be needed in the proof of our main theorems.

Lemma 2.1. [4] (i) Let $A$ be a maximal monotone operator. Then $\left(t^{-1} A\right)$ graph converges to $N_{A^{-1}}(0)$ as $t \rightarrow 0$ provided that $A^{-1}(0) \neq \emptyset$.

(ii) Let $\left\{A_{t}\right\}$ be a sequence of maximal monotone operators. If $B$ is a Lipschitz maximal monotone operator, then $A_{t}+B$ is maximal monotone. Furthermore, if $\left\{A_{t}\right\}$ graph converges to $A$, then $A$ is maximal monotone and $\left\{A_{t}+B\right\}$ graph converges to $A+B$.

Lemma 2.2. [2] Let $C$ be a nonempty closed convex subset of a real Hilbert space $H$ and let $T: C \rightarrow H$ be a nonexpansive mapping. Let $\left\{x_{n}\right\}$ be a sequence in $C$ and $x \in H$ such that $x_{n} \rightarrow x$ and $T x_{n}-x_{n} \rightarrow 0$ as $n \rightarrow+\infty$. Then $x \in \operatorname{Fix}(T)$.

Lemma 2.3. [24] Let $\left\{a_{n}\right\}$ be an iterative sequence of nonnegative real numbers such that $a_{n+1} \leq \mu_{n} \tau_{n}+v_{n}+\left(1-\mu_{n}\right) a_{n}$, where $\left\{\mu_{n}\right\}$ is a sequence in $(0,1)$ and $\left\{\tau_{n}\right\}$ is a real sequence. Assume that $\sum_{n=1}^{\infty} v_{n}<\infty$. Then, the following results hold:

(i) If $\tau_{n} \leq M$ for some $M \geq 0$, then $\left\{a_{n}\right\}$ is a bounded sequence.

(ii) If $\sum_{n=1}^{\infty} \mu_{n}=\infty$ and $\lim \sup _{n \rightarrow \infty} \tau_{n} \leq 0$, then $\lim _{n \rightarrow \infty} a_{n}=0$.

\section{The INERTIAL Viscosity ITERATIVE Algorithm}

Theorem 3.1. Let $C$ be a nonempty convex closed set in a real Hilbert space $H$. Let $f: C \rightarrow C$ be a $\rho$-contraction mapping with $\rho \in[0,1)$. Let $S, T: C \rightarrow C$ be two nonexpansive mappings. Let $\left\{v_{n}\right\}$ and $\left\{\psi_{n}\right\}$ be two sequences in $(0,1)$. Suppose that the following conditions are satisfied:

(P1) $\lim _{n \rightarrow \infty} v_{n}=0$ and $\lim _{n \rightarrow \infty} \frac{\psi_{n}}{v_{n}}=0$; 
(P2) $\lim _{n \rightarrow \infty} \psi_{n}=0$ and $\sum \psi_{n}=\infty$;

(P3) $\lim _{n \rightarrow \infty} \frac{v_{n}-v_{n-1}}{v_{n}^{2} \psi_{n}}=0$ and $\lim _{n \rightarrow \infty} \frac{\psi_{n}-\psi_{n-1}}{v_{n} \psi_{n}}=0$;

(P4) $\operatorname{Fix}(T) \cap \operatorname{int} C \neq \emptyset$;

(P5) $\lim _{n \rightarrow \infty} \frac{\delta_{n}\left\|x_{n}-x_{n-1}\right\|}{v_{n} \psi_{n}}=0$.

Set $x_{-1}, x_{0} \in C$ arbitrarily. Define a sequence $\left\{x_{n}\right\}$ by the following algorithm:

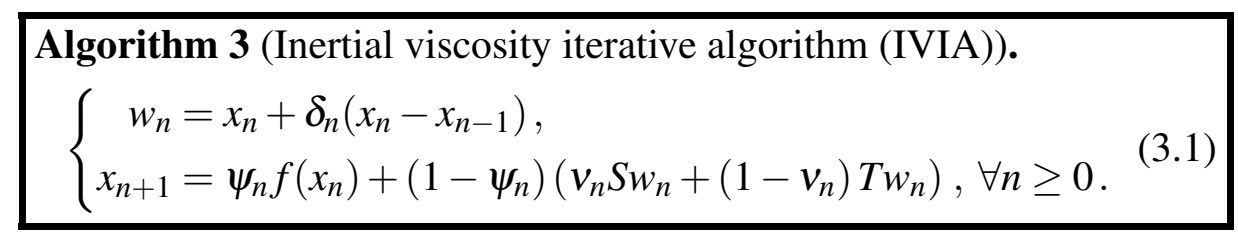

Then every weak cluster point of $\left\{x_{n}\right\}$ defined by (3.1) belongs to $\Omega$, the solution set of (HFPP).

Proof. First, we prove that the sequence $\left\{x_{n}\right\}$ is bounded. Take $u \in \operatorname{Fix}(T)$. From (3.1), we have

$$
\begin{aligned}
& \left\|x_{n+1}-u\right\| \\
\leq & \left\|\psi_{n}\left(f\left(x_{n}\right)-u\right)+\left(1-\psi_{n}\right)\left(v_{n}\left(S w_{n}-u\right)+\left(1-v_{n}\right)\left(T w_{n}-u\right)\right)\right\| \\
\leq & \psi_{n}\left\|f\left(x_{n}\right)-f(u)\right\|+\psi_{n}\|f(u)-u\|+\left(1-\psi_{n}\right)\left(v_{n}\left\|S w_{n}-u\right\|+\left(1-v_{n}\right)\left\|T w_{n}-u\right\|\right) \\
\leq & \psi_{n} \rho\left\|x_{n}-u\right\|+\psi_{n}\|f(u)-u\|+\left(1-\psi_{n}\right)\left\|w_{n}-u\right\| \\
\leq & \left(1-(1-\rho) \psi_{n}\right)\left\|x_{n}-u\right\|+(1-\rho) \psi_{n} \frac{\|f(u)-u\|}{1-\rho}+\left(1-\psi_{n}\right) \delta_{n}\left\|x_{n}-x_{n-1}\right\| .
\end{aligned}
$$

Let

$$
M:=2 \max \left\{\frac{\|f(u)-u\|}{1-\rho}, \sup _{n \geqslant 0} \frac{\left(1-\psi_{n}\right) \delta_{n}}{(1-\rho) \psi_{n}}\left\|x_{n}-x_{n-1}\right\|\right\} .
$$

From (3.2), one obtains

$$
\left\|x_{n+1}-u\right\| \leq\left(1-(1-\rho) \psi_{n}\right)\left\|x_{n}-u\right\|+(1-\rho) \psi_{n} M .
$$

From Lemma 2.3, one concludes that the sequence $\left\{x_{n}\right\}$ is bounded. From (3.1), one has

$$
\begin{aligned}
x_{n+1}-x_{n}= & \psi_{n}\left(f\left(x_{n}\right)-f\left(x_{n-1}\right)\right) \\
& +\left(1-\psi_{n}\right)\left(v_{n}\left(S w_{n}-S w_{n-1}\right)+\left(1-v_{n}\right)\left(T w_{n}-T w_{n-1}\right)\right) \\
& +\left(\psi_{n-1}-\psi_{n}\right)\left(-f\left(x_{n-1}\right)+v_{n-1} S w_{n-1}+\left(1-v_{n-1}\right) T w_{n-1}\right) \\
& +\left(1-\psi_{n}\right)\left(v_{n}-v_{n-1}\right)\left(S w_{n-1}-T w_{n-1}\right) .
\end{aligned}
$$

Therefore,

$$
\begin{aligned}
\left\|x_{n+1}-x_{n}\right\| \leq & \psi_{n} \rho\left\|x_{n}-x_{n-1}\right\|+\left(1-\psi_{n}\right)\left\|w_{n}-w_{n-1}\right\| \\
& +\left|\psi_{n-1}-\psi_{n}\right|\left\|-f\left(x_{n-1}\right)+v_{n-1} S w_{n-1}+\left(1-v_{n-1}\right) T w_{n-1}\right\| \\
& +\left(1-\psi_{n}\right)\left|v_{n}-v_{n-1}\right|\left\|S w_{n-1}-T w_{n-1}\right\| .
\end{aligned}
$$

Since $\left\{x_{n}\right\}$ is bounded, one concludes that $\left\{w_{n}\right\},\left\{f\left(x_{n}\right)\right\},\left\{S w_{n}\right\}$ and $\left\{T w_{n}\right\}$ are also bounded. Consequently, one infers that

$$
\begin{aligned}
\left\|x_{n+1}-x_{n}\right\| \leq & \left(1-(1-\rho) \psi_{n}\right)\left\|x_{n}-x_{n-1}\right\|+M_{1}\left(\left|\psi_{n-1}-\psi_{n}\right|+\left|v_{n}-v_{n-1}\right|\right) \\
& +\delta_{n}\left\|x_{n}-x_{n-1}\right\|+\delta_{n-1}\left\|x_{n-1}-x_{n-2}\right\|,
\end{aligned}
$$


where $M_{1}:=\sup _{n \geq 0}\left\{\left\|-f\left(x_{n-1}\right)+v_{n-1} S w_{n-1}+\left(1-v_{n-1}\right) T w_{n-1}\right\|,\left\|S w_{n-1}-T w_{n-1}\right\|\right\}$. From (3.3), one has

$$
\begin{aligned}
\frac{1}{v_{n}}\left\|x_{n+1}-x_{n}\right\| \leq & \left(1-(1-\rho) \psi_{n}\right)\left(\frac{1}{v_{n}}\left\|x_{n}-x_{n-1}\right\|\right)+M_{1}\left(\frac{\left|\psi_{n-1}-\psi_{n}\right|}{v_{n}}+\frac{\left|v_{n}-v_{n-1}\right|}{v_{n}}\right) \\
& +\frac{\delta_{n}}{v_{n}}\left\|x_{n}-x_{n-1}\right\|+\frac{\delta_{n-1}}{v_{n}}\left\|x_{n-1}-x_{n-2}\right\| \\
\leq & \left(1-(1-\rho) \psi_{n}\right)\left(\frac{1}{v_{n-1}}\left\|x_{n}-x_{n-1}\right\|\right)+M_{1}\left(\frac{\left|\psi_{n-1}-\psi_{n}\right|}{v_{n}}+\frac{\left|v_{n}-v_{n-1}\right|}{v_{n}}\right) \\
& +\left(1-(1-\rho) \psi_{n}\right)\left(\frac{1}{v_{n}}\left\|x_{n}-x_{n-1}\right\|-\frac{1}{v_{n-1}}|| x_{n}-x_{n-1} \|\right) \\
& +\frac{\delta_{n}}{v_{n}}\left\|x_{n}-x_{n-1}\right\|+\frac{\delta_{n-1}}{v_{n}}\left\|x_{n-1}-x_{n-2}\right\| \\
\leq & \left(1-(1-\rho) \psi_{n}\right)\left(\frac{1}{v_{n-1}}\left\|x_{n}-x_{n-1}\right\|\right)+(1-\rho) \psi_{n}\left(\frac{1}{\psi_{n}}\left|\frac{1}{v_{n}}-\frac{1}{v_{n-1}}\right|\right. \\
& \left.+\frac{1}{v_{n} \psi_{n}}\left|v_{n}-v_{n-1}\right|+\frac{1}{v_{n} \psi_{n}}\left|\psi_{n}-\psi_{n-1}\right|\right) \frac{M_{2}}{(1-\rho)} \\
& +(1-\rho) \psi_{n}\left(\frac{\delta_{n}}{(1-\rho) v_{n} \psi_{n}}\left\|x_{n}-x_{n-1}\right\|+\frac{\delta_{n-1}}{(1-\rho) v_{n} \psi_{n}}\left\|x_{n-1}-x_{n-2}\right\|\right),
\end{aligned}
$$

where $M_{2}$ is a positive constant such that $\sup _{n \geq 0}\left\{\left\|x_{n}-x_{n-1}\right\|, M_{1}\right\} \leq M_{2}$. Using (P1), (P2), (P3) and (P5), in the light of Lemma 2.3, we deduce that

$$
\frac{1}{v_{n}}\left\|x_{n+1}-x_{n}\right\| \rightarrow 0, \text { as } n \rightarrow \infty \text {. }
$$

Thus, $\left\|x_{n+1}-x_{n}\right\| \rightarrow 0$. By using (3.1), (P1) and (P2), one concludes that $\left\|x_{n+1}-T w_{n}\right\| \rightarrow 0$. Therefore, $\left\|x_{n}-T w_{n}\right\| \leq\left\|x_{n}-x_{n+1}\right\|+\left\|x_{n+1}-T w_{n}\right\| \rightarrow 0$ as $n \rightarrow \infty$. By the definition of $w_{n}$ and (P5), one has $\left\|w_{n}-x_{n}\right\| \rightarrow 0$ as $n \rightarrow \infty$. Thus,

$$
\begin{aligned}
\left\|x_{n}-T x_{n}\right\| & \leq\left\|x_{n}-T w_{n}\right\|+\left\|T w_{n}-T x_{n}\right\| \\
& \leq\left\|x_{n}-T w_{n}\right\|+\left\|w_{n}-x_{n}\right\| \rightarrow 0, \text { as } n \rightarrow \infty .
\end{aligned}
$$

Without loss of the generality, one may assumes that $\left\{x_{n}\right\} \rightarrow x^{*}$. Combining (3.5) and Lemma 2.2, we see that $x^{*} \in \operatorname{Fix}(T)$. Again by using (3.1), one obtains

$$
x_{n+1}-w_{n}=\psi_{n}\left(f\left(x_{n}\right)-w_{n}\right)+\left(1-\psi_{n}\right)\left(v_{n}\left(S w_{n}-w_{n}\right)+\left(1-v_{n}\right)\left(T w_{n}-w_{n}\right)\right),
$$

that is,

$$
\frac{w_{n}-x_{n+1}}{\left(1-\psi_{n}\right) v_{n}}+\frac{\psi_{n} \delta_{n}\left(x_{n}-x_{n-1}\right)}{\left(1-\psi_{n}\right) v_{n}}=\frac{\psi_{n}}{\left(1-\psi_{n}\right) v_{n}}(I-f) x_{n}+(I-S) w_{n}+\frac{1-v_{n}}{v_{n}}(I-T) w_{n} .
$$

Lemma 2.1 assures that the operator sequence $\left\{\frac{1-v_{n}}{v_{n}}(I-T)\right\}$ graph converges to $N_{\operatorname{Fix}(T)}$ and $\left\{\frac{\psi_{n}}{\left(1-\psi_{n}\right) v_{n}}(I-f)\right\}$ graph converges to $N_{C}$, which allows us to infer, in the light of a result in [1], that operator $(I-S)+\frac{1-v_{n}}{v_{n}}(I-T)+\frac{\psi_{n}}{\left(1-\psi_{n}\right) v_{n}}(I-f)$ graph converges to $(I-S)+N_{C}+$ $N_{\text {Fix }(T)}$. The latter coincides with $(I-S)+N_{\text {Fix }(T)}$ thanks to the condition (P4). Since $\frac{w_{n}-x_{n+1}}{\left(1-\psi_{n}\right) v_{n}}+$ $\frac{\psi_{n} \delta_{n}\left(x_{n}-x_{n-1}\right)}{\left(1-\psi_{n}\right) v_{n}} \rightarrow 0$ and the graph of $(I-S)+N_{\operatorname{Fix}(T)}$ is weakly-strongly closed, we finally obtain $0 \in(I-S) x^{*}+N_{\text {Fix }(T)} x^{*}$, that is, $x^{*}$ solves problem (HFPP). 
Remark 3.1. (i) We remark here that condition (P5) is easily implemented in numerical computation since the value of $\left\|x_{n}-x_{n-1}\right\|$ is known before choosing $\delta_{n}$. Therefore, parameter $\delta_{n}$ can be chosen such that $0 \leq \delta_{n} \leq \hat{\delta}_{n}$, where

$$
\hat{\delta}_{n}= \begin{cases}\min \left\{\frac{\xi_{n}}{\left\|x_{n}-x_{n-1}\right\|}, \delta\right\}, & \text { if } x_{n} \neq x_{n-1} ; \\ \delta, & \text { otherwise }\end{cases}
$$

and $\left\{\xi_{n}\right\}$ is a positive sequence such that $\lim _{n \rightarrow \infty} \frac{\xi_{n}}{v_{n} \psi_{n}}=0$.

(ii) Prototypes for the iterative parameters are, for example, $v_{n}=\frac{1}{n^{a}}, \psi_{n}=\frac{1}{n^{b}}$ and $\xi_{n}=\frac{1}{n^{c}}$ provided that $a \in(0,1 / 2), b \in(a, 1-a)$ and $c \in(a+b, \infty)$.

Theorem 3.2. Let $C$ be a nonempty convex closed set in a real Hilbert space $H$. Let $f: C \rightarrow C$ be a $\rho$-contraction mapping with $\rho \in[0,1)$. Let $S, T: C \rightarrow C$ be two nonexpansive mappings. Let $\left\{v_{n}\right\}$ and $\left\{\psi_{n}\right\}$ be two sequences in $(0,1)$. Suppose that the following conditions are satisfied:

(P1) $\lim _{n \rightarrow \infty} v_{n}=0$ and $\lim _{n \rightarrow \infty} \frac{\psi_{n}}{v_{n}}=0$;

(P2) $\lim _{n \rightarrow \infty} \psi_{n}=0$ and $\sum \psi_{n}=\infty$;

(P3) $\lim _{n \rightarrow \infty} \frac{v_{n}-v_{n-1}}{v_{n}^{2} \psi_{n}}=0$ and $\lim _{n \rightarrow \infty} \frac{\psi_{n}-\psi_{n-1}}{v_{n} \psi_{n}}=0$;

(P4) $\operatorname{Fix}(T) \cap \operatorname{int} C \neq \emptyset$;

(P5) $\lim _{n \rightarrow \infty} \frac{\delta_{n}\left\|x_{n}-x_{n-1}\right\|}{v_{n} \psi_{n}}=0$;

(P6) There exist two positive constants $k$ and $\lambda$ such that $\|x-T x\| \geq k \operatorname{Dist}(x, \operatorname{Fix}(T))^{\lambda}$, where $\operatorname{Dist}(x, \operatorname{Fix}(T)):=\inf _{y \in \operatorname{Fix}(T)}\|y-x\| ;$

(P7) $\lim _{n \rightarrow \infty} \frac{v_{n}^{1+1 / \lambda}}{\psi_{n}}=0$.

Then the sequence $\left\{x_{n}\right\}$ generated by (3.1) converges to $x^{*}=\mathrm{P}_{\Omega} f\left(x^{*}\right)$ in norm.

Proof. From (3.1), we can write

$$
\begin{aligned}
x_{n+1}-x^{*}= & \psi_{n}\left(f\left(x_{n}\right)-x^{*}\right)+\left(1-\psi_{n}\right)\left(v_{n}\left(S w_{n}-x^{*}\right)+\left(1-v_{n}\right)\left(T w_{n}-x^{*}\right)\right) \\
= & \left(\psi_{n}\left(f\left(x_{n}\right)-f\left(x^{*}\right)\right)+\left(1-\psi_{n}\right)\left(v_{n}\left(S w_{n}-S x^{*}\right)+\left(1-v_{n}\right)\left(T w_{n}-x^{*}\right)\right)\right) \\
& +\left(\psi_{n}\left(f\left(x^{*}\right)-x^{*}\right)+v_{n}\left(1-\psi_{n}\right)\left(S x^{*}-x^{*}\right)\right) .
\end{aligned}
$$

An elementary computation yields $\|a+b\|^{2}-2\langle b, a+b\rangle=\|a\|^{2}-\|b\|^{2}, \forall a, b \in H$. It follows that

$$
\begin{gathered}
\left\|x_{n+1}-x^{*}\right\|^{2}-2\left\langle\psi_{n}\left(f\left(x^{*}\right)-x^{*}\right)+v_{n}\left(1-\psi_{n}\right)\left(S x^{*}-x^{*}\right), x_{n+1}-x^{*}\right\rangle \\
\leq\left\|\psi_{n}\left(f\left(x_{n}\right)-f\left(x^{*}\right)\right)+\left(1-\psi_{n}\right)\left(v_{n}\left(S w_{n}-S x^{*}\right)+\left(1-v_{n}\right)\left(T w_{n}-x^{*}\right)\right)\right\|^{2} .
\end{gathered}
$$

By convexity of the mapping $x \rightarrow\|x\|^{2}$, one sees that

$$
\begin{aligned}
& \left\|x_{n+1}-x^{*}\right\|^{2}-2\left\langle\psi_{n}\left(f\left(x^{*}\right)-x^{*}\right)+v_{n}\left(1-\psi_{n}\right)\left(S x^{*}-x^{*}\right), x_{n+1}-x^{*}\right\rangle \\
\leq & \psi_{n}\left\|f\left(x_{n}\right)-f\left(x^{*}\right)\right\|^{2}+\left(1-\psi_{n}\right)\left\|v_{n}\left(S w_{n}-S x^{*}\right)+\left(1-v_{n}\right)\left(T w_{n}-x^{*}\right)\right\|^{2} \\
\leq & \psi_{n}\left\|f\left(x_{n}\right)-f\left(x^{*}\right)\right\|^{2}+\left(1-\psi_{n}\right)\left(v_{n}\left\|S w_{n}-S x^{*}\right\|^{2}+\left(1-v_{n}\right)\left\|T w_{n}-x^{*}\right\|^{2}\right) \\
\leq & \psi_{n} \rho^{2}\left\|x_{n}-x^{*}\right\|^{2}+\left(1-\psi_{n}\right)\left(v_{n}\left\|w_{n}-x^{*}\right\|^{2}+\left(1-v_{n}\right)\left\|w_{n}-x^{*}\right\|^{2}\right) \\
\leq & \left(1-\left(1-\rho^{2}\right) \psi_{n}\right)\left\|x_{n}-x^{*}\right\|^{2}+2 \delta_{n}\left\langle x_{n}-x_{n-1}, w_{n}-x^{*}\right\rangle,
\end{aligned}
$$


which yields

$$
\begin{aligned}
\left\|x_{n+1}-x^{*}\right\|^{2} \leq & \left(1-\left(1-\rho^{2}\right) \psi_{n}\right)\left\|x_{n}-x^{*}\right\|^{2}+2 \delta_{n}\left\langle x_{n}-x_{n-1}, w_{n}-x^{*}\right\rangle \\
& +2 \psi_{n}\left\langle f\left(x^{*}\right)-x^{*}, x_{n+1}-x^{*}\right\rangle+2 v_{n}\left(1-\psi_{n}\right)\left\langle S x^{*}-x^{*}, x_{n+1}-x^{*}\right\rangle .
\end{aligned}
$$

From condition (P5) and the fact that $\left\{w_{n}\right\}$ is bounded, we have

$$
\limsup _{n \rightarrow \infty} 2 \delta_{n}\left\langle x_{n}-x_{n-1}, w_{n}-x^{*}\right\rangle=0 .
$$

By Theorem 3.1, we have that any weak cluster point of $\left\{x_{n}\right\}$ is in $\Omega$. Therefore, it is easy conclude from $x^{*}=P_{\Omega} f\left(x^{*}\right)$ that

$$
\limsup _{n \rightarrow \infty}\left\langle f\left(x^{*}\right)-x^{*}, x_{n}-x^{*}\right\rangle \leq 0 .
$$

On the other hand, we have

$$
\left\langle S x^{*}-x^{*}, x_{n+1}-x^{*}\right\rangle=\left\langle S x^{*}-x^{*}, P_{\operatorname{Fix}(T)} x_{n+1}-x^{*}\right\rangle+\left\langle S x^{*}-x^{*}, x_{n+1}-P_{\operatorname{Fix}(T)} x_{n+1}\right\rangle .
$$

Since $P_{\operatorname{Fix}(T)} x_{n+1} \in \operatorname{Fix}(T)$, according to (HFPP), we obtain

$$
\left\langle S x^{*}-x^{*}, P_{\operatorname{Fix}(T)} x_{n+1}-x^{*}\right\rangle \leq 0,
$$

which further yields that

$$
\begin{aligned}
\left\langle S x^{*}-x^{*}, x_{n+1}-x^{*}\right\rangle & \leq\left\langle S x^{*}-x^{*}, x_{n+1}-P_{\operatorname{Fix}(T)^{x}} x_{n+1}\right\rangle \\
& \leq\left\|S x^{*}-x^{*}\right\|\left\|x_{n+1}-P_{\operatorname{Fix}(T)^{x}} x_{n+1}\right\| \\
& =\left\|S x^{*}-x^{*}\right\| \operatorname{Dist}\left(x_{n+1}, \operatorname{Fix}(T)\right) .
\end{aligned}
$$

Consequently, one concludes from (P6) that

$$
\left\langle S x^{*}-x^{*}, x_{n+1}-x^{*}\right\rangle \leq k^{-1 / \lambda}\left\|S x^{*}-x^{*}\right\|\left\|T x_{n+1}-x_{n+1}\right\|^{1 / \lambda} .
$$

In view of (3.1), we have

$$
\begin{aligned}
\left\|x_{n+1}-T x_{n+1}\right\| & \leq \psi_{n}\left\|f\left(x_{n}\right)\right\|+v_{n}\left\|S w_{n}-T w_{n}\right\|+\left\|x_{n+1}-w_{n}\right\| \\
& \leq k_{1}\left(v_{n}+\psi_{n}+\left\|x_{n+1}-w_{n}\right\|\right),
\end{aligned}
$$

where $k_{1}:=\sup _{n \geq 0}\left\{\left\|f\left(x_{n}\right)\right\|,\left\|S w_{n}-T w_{n}\right\|, 1\right\}$. This together with (3.10) implies that

$$
\left\langle S x^{*}-x^{*}, x_{n+1}-x^{*}\right\rangle \leq k_{2}\left(v_{n}+\psi_{n}+\left\|x_{n+1}-w_{n}\right\|\right)^{1 / \lambda}
$$

for a positive constant $k_{2}:=k_{1}^{1 / \lambda} k^{-1 / \lambda}\left\|S x^{*}-x^{*}\right\|$.

Now, in the light of the assumptions (P1), (P5), (P7) and (3.4), we obtain

$$
\begin{aligned}
\lim _{n \rightarrow \infty} \frac{v_{n}}{\psi_{n}}\left(v_{n}+\psi_{n}+\left\|x_{n+1}-w_{n}\right\|\right)^{1 / \lambda} & =\lim _{n \rightarrow \infty} \frac{v_{n}^{1+1 / \lambda}}{\psi_{n}}\left(1+\frac{\psi_{n}}{v_{n}}+\frac{\left\|x_{n+1}-w_{n}\right\|}{v_{n}}\right)^{1 / \lambda} \\
& =\lim _{n \rightarrow \infty} \frac{v_{n}^{1+1 / \lambda}}{\psi_{n}}=0,
\end{aligned}
$$

which by (3.11) leads to

$$
\limsup _{n \rightarrow \infty} \frac{v_{n}}{\psi_{n}}\left\langle S x^{*}-x^{*}, x_{n+1}-x^{*}\right\rangle \leq 0 .
$$

Finally, by (3.7), (3.8), (3.9), (3.12) and using Lemma 2.3, we conclude that the sequence $\left\{x_{n}\right\}$ converges to $x^{*}$ in norm. This completes the proof. 
Remark 3.2. (i) Prototypes for the iterative parameters are, for instance, $v_{n}=\frac{1}{n^{a}}, \psi_{n}=\frac{1}{n^{b}}$ and $\xi_{n}=\frac{1}{n^{c}}$ provided that $a \in\left(0, \frac{1}{2+1 / \lambda}\right], b \in(a, a(1+1 / \lambda))$ and $c \in(a+b, \infty)$ or $a \in\left(\frac{1}{2+1 / \lambda}, 1 / 2\right), b \in(a, 1-a)$ and $c \in(a+b, \infty)$.

(ii) When $\delta_{n}=0$ in (3.1), then Algorithm IVIA (3.1) becomes the Algorithm VIA (1.2) proposed by Mainge and Moudafi [13].

\section{THE INERTIAL TWO-STEP ITERATIVE ALGORITHM}

Theorem 4.1. Let $C$ be a nonempty convex closed set in a real Hilbert space $H$. Let $f: C \rightarrow C$ be a $\rho$-contraction mapping with $\rho \in[0,1)$. Let $S, T: C \rightarrow C$ be two nonexpansive mappings. Let $\left\{v_{n}\right\}$ and $\left\{\psi_{n}\right\}$ be two sequences in $(0,1)$. Suppose that the following conditions are satisfied:

(C1) $\lim _{n \rightarrow \infty} \psi_{n}=0$ and $\sum_{n=1}^{\infty} \psi_{n}=\infty$;

(C2) $\lim _{n \rightarrow \infty} v_{n}=0$ and $\lim _{n \rightarrow \infty} \frac{\psi_{n}}{v_{n}}=0$;

(C3) $\lim _{n \rightarrow \infty} \frac{1}{\psi_{n}}\left|\frac{1}{v_{n}}-\frac{1}{v_{n-1}}\right|=0$ and $\lim _{n \rightarrow \infty} \frac{1}{v_{n}}\left|1-\frac{\psi_{n-1}}{\psi_{n}}\right|=0$;

(C4) $\operatorname{Fix}(T) \cap \operatorname{int} C \neq \emptyset$;

(C5) $\lim _{n \rightarrow \infty} \frac{\delta_{n}\left\|x_{n}-x_{n-1}\right\|}{v_{n} \psi_{n}}=0$.

Set $x_{-1}, x_{0} \in C$ arbitrarily. Define a sequence $\left\{x_{n}\right\}$ by the following algorithm:

Algorithm 4 (Inertial Two-step iterative algorithm (ITWIA)).
\[ \begin{array}{c}w_{n}=x_{n}+\delta_{n}\left(x_{n}-x_{n-1}\right) \\ y_{n}=v_{n} S w_{n}+\left(1-v_{n}\right) w_{n} \\ x_{n+1}=\psi_{n} f\left(x_{n}\right)+\left(1-\psi_{n}\right) T y_{n}, \quad \forall n \geq 0\end{array} \]

Then every weak cluster point of $\left\{x_{n}\right\}$ created by (4.1) belongs to the solution set $\Omega$ of (HFPP).

Proof. First, we prove that the sequence $\left\{x_{n}\right\}$ is bounded. Take $u \in \operatorname{Fix}(T)$. From (4.1), we have $\left\|w_{n}-u\right\| \leq\left\|x_{n}-u\right\|+\delta_{n}\left\|x_{n}-x_{n-1}\right\|$, and

$$
\begin{aligned}
& \left\|x_{n+1}-u\right\| \\
\leq & \left\|\psi_{n} f\left(x_{n}\right)+\left(1-\psi_{n}\right) T y_{n}-u\right\| \\
\leq & \psi_{n}\left\|f\left(x_{n}\right)-f(u)\right\|+\psi_{n}\|f(u)-u\|+\left(1-\psi_{n}\right)\left\|T y_{n}-u\right\| \\
\leq & \psi_{n} \rho\left\|x_{n}-u\right\|+\psi_{n}\|f(u)-u\|+\left(1-\psi_{n}\right)\left\|y_{n}-u\right\| \\
\leq & \psi_{n} \rho\left\|x_{n}-u\right\|+\psi_{n}\|f(u)-u\|+\left(1-\psi_{n}\right) v_{n}\left\|S w_{n}-u\right\|+\left(1-\psi_{n}\right)\left(1-v_{n}\right)\left\|w_{n}-u\right\| \\
\leq & \psi_{n} \rho\left\|x_{n}-u\right\|+\psi_{n}\|f(u)-u\|+\left(1-\psi_{n}\right)\left\|w_{n}-u\right\| \\
= & \left(1-(1-\rho) \psi_{n}\right)\left\|x_{n}-u\right\|+(1-\rho) \psi_{n} \frac{\|f(u)-u\|}{1-\rho}+\left(1-\psi_{n}\right) \delta_{n}\left\|x_{n}-x_{n-1}\right\| .
\end{aligned}
$$

Let

$$
M:=2 \max \left\{\frac{\|f(u)-u\|}{1-\rho}, \sup _{n \geqslant 0} \frac{\left(1-\psi_{n}\right) \delta_{n}}{(1-\rho) \psi_{n}}\left\|x_{n}-x_{n-1}\right\|\right\} .
$$

Using (4.2), one has

$$
\left\|x_{n+1}-u\right\| \leq\left(1-(1-\rho) \psi_{n}\right)\left\|x_{n}-u\right\|+(1-\rho) \psi_{n} M .
$$


By Lemma 2.3, we get that the sequence $\left\{x_{n}\right\}$ is bounded and hence the sequences $\left\{f\left(x_{n}\right)\right\}$, $\left\{y_{n}\right\},\left\{T x_{n}\right\}$ and $\left\{T y_{n}\right\}$ are also bounded. From (4.1), we obtain

$$
\begin{aligned}
\left\|x_{n+1}-x_{n}\right\| \leq & \psi_{n}\left\|f\left(x_{n}\right)-f\left(x_{n-1}\right)\right\|+\left|\psi_{n}-\psi_{n-1}\right|\left\|f\left(x_{n-1}\right)\right\| \\
& +\left(1-\psi_{n}\right)\left\|T y_{n}-T y_{n-1}\right\|+\left|\psi_{n}-\psi_{n-1}\right|\left\|T y_{n-1}\right\| \\
\leq & \rho \psi_{n}\left\|x_{n}-x_{n-1}\right\|+\left(1-\psi_{n}\right)\left\|y_{n}-y_{n-1}\right\| \\
& +\left|\psi_{n}-\psi_{n-1}\right|\left(\left\|f\left(x_{n-1}\right)\right\|+\left\|T y_{n-1}\right\|\right) .
\end{aligned}
$$

By using (4.1), one also has

$$
\begin{aligned}
\left\|y_{n}-y_{n-1}\right\| \leq & v_{n}\left\|S w_{n}-S w_{n-1}\right\|+\left(1-v_{n}\right)\left\|w_{n}-w_{n-1}\right\| \\
& +\left|v_{n}-v_{n-1}\right|\left(\left\|S w_{n-1}\right\|+\left\|w_{n-1}\right\|\right) \\
\leq & \left\|w_{n}-w_{n-1}\right\|+\left|v_{n}-v_{n-1}\right|\left(\left\|S w_{n-1}\right\|+\left\|w_{n-1}\right\|\right) .
\end{aligned}
$$

Combining (4.3) and (4.4), we find that

$$
\begin{aligned}
& \frac{\left\|x_{n+1}-x_{n}\right\|}{v_{n}} \\
\leq & \left(1-(1-\rho) \psi_{n}\right) \frac{\left\|x_{n}-x_{n-1}\right\|}{v_{n}}+\frac{\left|v_{n}-v_{n-1}\right|}{v_{n}}\left(\left\|S w_{n-1}\right\|+\left\|w_{n-1}\right\|\right) \\
& +\frac{\left|\psi_{n}-\psi_{n-1}\right|}{v_{n}}\left(\left\|f\left(x_{n-1}\right)\right\|+\left\|T y_{n-1}\right\|\right)+\frac{\delta_{n}}{v_{n}}\left\|x_{n}-x_{n-1}\right\|+\frac{\delta_{n-1}}{v_{n}}\left\|x_{n-1}-x_{n-2}\right\| \\
= & \left(1-(1-\rho) \psi_{n}\right) \frac{\left\|x_{n}-x_{n-1}\right\|}{v_{n-1}}+\left(1-(1-\rho) \psi_{n}\right)\left(\frac{\left\|x_{n}-x_{n-1}\right\|}{v_{n}}-\frac{\left\|x_{n}-x_{n-1}\right\|}{v_{n-1}}\right) \\
& +\frac{\left|v_{n}-v_{n-1}\right|}{v_{n}}\left(\left\|S w_{n-1}\right\|+\left\|w_{n-1}\right\|\right)+\frac{\left|\psi_{n}-\psi_{n-1}\right|}{v_{n}}\left(\left\|f\left(x_{n-1}\right)\right\|+\left\|T y_{n-1}\right\|\right) \\
& +\frac{\delta_{n}}{v_{n}}\left\|x_{n}-x_{n-1}\right\|+\frac{\delta_{n-1}}{v_{n}}\left\|x_{n-1}-x_{n-2}\right\| \\
\leq & \left(1-(1-\rho) \psi_{n}\right) \frac{\left\|x_{n}-x_{n-1}\right\|}{v_{n-1}}+\left(\left|\frac{1}{v_{n}}-\frac{1}{v_{n-1}}\right|+\frac{\left|v_{n}-v_{n-1}\right|}{v_{n}}+\frac{\left|\psi_{n}-\psi_{n-1}\right|}{v_{n}}\right) M_{1} \\
& +\frac{\delta_{n}}{v_{n}}\left\|x_{n}-x_{n-1}\right\|+\frac{\delta_{n-1}}{v_{n}}\left\|x_{n-1}-x_{n-2}\right\| \\
= & \left(1-(1-\rho) \psi_{n}\right) \frac{\left\|x_{n}-x_{n-1}\right\|}{v_{n-1}}+(1-\rho) \psi_{n}\left(\frac{1}{\psi_{n}}\left|\frac{1}{v_{n}}-\frac{1}{v_{n-1}}\right|\right. \\
& \left.+\frac{1}{\psi_{n}} \frac{\left|v_{n}-v_{n-1}\right|}{v_{n}}+\frac{1}{\psi_{n}} \frac{\left|\psi_{n}-\psi_{n-1}\right|}{v_{n}}\right) \frac{M_{1}}{1-\rho} \\
& +(1-\rho) \psi_{n}\left(\frac{\delta_{n}}{(1-\rho) v_{n} \psi_{n}}\left\|x_{n}-x_{n-1}\right\|+\frac{\delta_{n-1}}{(1-\rho) v_{n} \psi_{n}}\left\|x_{n-1}-x_{n-2}\right\|\right),
\end{aligned}
$$

where $M_{1}$ satisfies

$$
\sup _{n \geq 0}\left\{\left\|x_{n}-x_{n-1}\right\|,\left(\left\|S w_{n-1}\right\|+\left\|w_{n-1}\right\|\right),\left(\left\|f\left(x_{n-1}\right)\right\|+\left\|T y_{n-1}\right\|\right)\right\} \leq M_{1} .
$$

From (C3), we observe that $\lim _{n \rightarrow \infty} \frac{1}{v_{n-1}}\left|\frac{v_{n}-v_{n-1}}{\psi_{n} v_{n}}\right|=0$ implies

$$
\lim _{n \rightarrow \infty} \frac{1}{\psi_{n}} \frac{\left|v_{n}-v_{n-1}\right|}{v_{n}}=0 \text {. }
$$


Thus, from (C3) and (4.6), one obtains

$$
\lim _{n \rightarrow \infty}\left(\frac{1}{\psi_{n}}\left|\frac{1}{v_{n}}-\frac{1}{v_{n-1}}\right|+\frac{1}{\psi_{n}} \frac{\left|v_{n}-v_{n-1}\right|}{v_{n}}+\frac{1}{\psi_{n}} \frac{\left|\psi_{n}-\psi_{n-1}\right|}{v_{n}}\right)=0 .
$$

Hence, combining condition (C5), and applying Lemma 2.3 to (4.5), we immediately conclude that $\lim _{n \rightarrow \infty} \frac{\left\|x_{n+1}-x_{n}\right\|}{v_{n}}=0$, which leads us to

$$
\lim _{n \rightarrow \infty}\left\|x_{n+1}-x_{n}\right\|=0 .
$$

By (4.1), one has $\left\|x_{n+1}-T y_{n}\right\|=\psi_{n}\left\|f\left(x_{n}\right)-T y_{n}\right\| \rightarrow 0$ as $n \rightarrow \infty$, which together with (4.7) leads to $\left\|x_{n}-T y_{n}\right\| \leq\left\|x_{n+1}-x_{n}\right\|+\left\|x_{n+1}-T y_{n}\right\| \rightarrow 0$ as $n \rightarrow \infty$. Using (4.1) again, one concludes that $\left\|y_{n}-w_{n}\right\|=v_{n}\left\|S w_{n}-w_{n}\right\| \rightarrow 0$ as $n \rightarrow \infty$. By the definition of $w_{n}$ and condition (C5), one has $\left\|w_{n}-x_{n}\right\|=\delta_{n}\left\|x_{n}-x_{n-1}\right\| \rightarrow 0$ as $n \rightarrow \infty$. Hence,

$$
\left\|y_{n}-T y_{n}\right\| \leq\left\|y_{n}-w_{n}\right\|+\left\|w_{n}-x_{n}\right\|+\left\|x_{n}-T y_{n}\right\| \rightarrow 0, \text { as } n \rightarrow \infty .
$$

Since the sequence $\left\{y_{n}\right\}$ is bounded, there exists a subsequence of $\left\{y_{n}\right\}$, still denoted by $\left\{y_{n}\right\}$, which converges weakly to some $p \in H$. Therefore, $p \in \operatorname{Fix}(T)$ by (4.8) and the demiclosed principle. From (4.1), we observe that

$$
x_{n+1}-w_{n}=\psi_{n}\left(f\left(x_{n}\right)-w_{n}\right)+\left(1-\psi_{n}\right)\left(T y_{n}-y_{n}\right)+\left(1-\psi_{n}\right) v_{n}\left(S w_{n}-w_{n}\right),
$$

that is,

$$
\frac{w_{n}-x_{n+1}}{v_{n}\left(1-\psi_{n}\right)}+\frac{\psi_{n} \delta_{n}\left(x_{n}-x_{n-1}\right)}{v_{n}\left(1-\psi_{n}\right)}=\frac{\psi_{n}}{v_{n}\left(1-\psi_{n}\right)}(I-f) x_{n}+\frac{1}{v_{n}}(I-T) y_{n}+(I-S) w_{n} .
$$

Lemma 2.1 assures that the operator sequence $\left\{\frac{1}{v_{n}}(I-T)\right\}$ graph converges to $N_{\mathrm{Fix}(T)}$ and $\left\{\frac{\psi_{n}}{v_{n}\left(1-\psi_{n}\right)}(I-f)\right\}$ graph converges to $N_{C}$, which in the light of a result in [1] allows us to infer that the operator $(I-S)+\frac{\psi_{n}}{v_{n}\left(1-\psi_{n}\right)}(I-f)+\frac{1}{v_{n}}(I-T)$ graph converges to $(I-S)+N_{C}+N_{\text {Fix }}(T)$. This together with condition (C4) implies that $(I-S)+\frac{\psi_{n}}{v_{n}\left(1-\psi_{n}\right)}(I-f)+\frac{1}{v_{n}}(I-T)$ graph converges to $(I-S)+N_{\mathrm{Fix}(T)}$. Since $\frac{w_{n}-x_{n+1}}{v_{n}\left(1-\psi_{n}\right)}+\frac{\psi_{n} \delta_{n}\left(x_{n}-x_{n-1}\right)}{v_{n}\left(1-\psi_{n}\right)} \rightarrow 0$ and the graph $(I-S)+N_{\mathrm{Fix}(T)}$ is weakly-strongly closed, we obtain $0 \in(I-S) p+N_{\mathrm{Fix}(T)} p$. That is, $p$ solves the (HFPP).

Remark 4.1. Prototypes for the iterative parameters are, for example, $v_{n}=\frac{1}{n^{a}}, \psi_{n}=\frac{1}{n^{b}}$ and $\xi_{n}=\frac{1}{n^{c}}$, where $a \in\left(0, \frac{1}{2}\right), b \in(a, 1-a)$ and $c \in(a+b, \infty)$.

Theorem 4.2. Let $C$ be a nonempty convex closed set in a real Hilbert space $H$. Let $f: C \rightarrow C$ be a $\rho$-contraction mapping with $\rho \in[0,1)$. Let $S, T: C \rightarrow C$ be two nonexpansive mappings. Let $\left\{v_{n}\right\}$ and $\left\{\psi_{n}\right\}$ be two sequences in $(0,1)$. Suppose that the following conditions are satisfied:

(C1) $\lim _{n \rightarrow \infty} \psi_{n}=0$ and $\sum_{n=1}^{\infty} \psi_{n}=\infty$;

(C2) $\lim _{n \rightarrow \infty} v_{n}=0, \lim _{n \rightarrow \infty} \frac{\psi_{n}}{v_{n}}=0$ and $\lim _{n \rightarrow \infty} \frac{v_{n}^{2}}{\psi_{n}}=0$;

(C3) $\lim _{n \rightarrow \infty} \frac{1}{\psi_{n}}\left|\frac{1}{v_{n}}-\frac{1}{v_{n-1}}\right|=0$ and $\lim _{n \rightarrow \infty} \frac{1}{v_{n}}\left|1-\frac{\psi_{n-1}}{\psi_{n}}\right|=0$;

(C4) $\operatorname{Fix}(T) \cap \operatorname{int} C \neq \emptyset$;

(C5) $\lim _{n \rightarrow \infty} \frac{\delta_{n}\left\|x_{n}-x_{n-1}\right\|}{v_{n} \psi_{n}}=0$;

(C6) There exists a constant $k>0$ such that $\|x-T x\| \geq k \operatorname{Dist}(x, \operatorname{Fix}(T))$ for each $x \in C$, where $\operatorname{Dist}(x, \operatorname{Fix}(T))=\inf _{y \in \operatorname{Fix}(T)}\|x-y\|$.

Then the sequence $\left\{x_{n}\right\}$ formed by (4.1) converges to $p=\mathrm{P}_{\Omega} f(p)$ in norm. 
Proof. Take $p \in \operatorname{Fix}(T)$. From (4.1), we have

$$
x_{n+1}-p=\psi_{n}\left(f\left(x_{n}\right)-f(p)\right)+\psi_{n}(f(p)-p)+\left(1-\psi_{n}\right)\left(T y_{n}-p\right) .
$$

Thus,

$$
\begin{aligned}
\left\|x_{n+1}-p\right\|^{2} & \leq\left\|\psi_{n}\left(f\left(x_{n}\right)-f(p)\right)+\left(1-\psi_{n}\right)\left(T y_{n}-p\right)\right\|^{2}+2 \psi_{n}\left\langle f(p)-p, x_{n+1}-p\right\rangle \\
& \leq\left(1-\psi_{n}\right)\left\|T y_{n}-p\right\|^{2}+\psi_{n}\left\|f\left(x_{n}\right)-f(p)\right\|^{2}+2 \psi_{n}\left\langle f(p)-p, x_{n+1}-p\right\rangle \\
& \leq\left(1-\psi_{n}\right)\left\|y_{n}-p\right\|^{2}+\rho^{2} \psi_{n}\left\|x_{n}-p\right\|^{2}+2 \psi_{n}\left\langle f(p)-p, x_{n+1}-p\right\rangle .
\end{aligned}
$$

Using (4.1), we obtain

$$
\begin{aligned}
\left\|y_{n}-p\right\|^{2} & =\left\|\left(1-v_{n}\right)\left(w_{n}-p\right)+v_{n}\left(S w_{n}-S p\right)+v_{n}(S p-p)\right\|^{2} \\
& \leq\left\|\left(1-v_{n}\right)\left(w_{n}-p\right)+v_{n}\left(S w_{n}-S p\right)\right\|^{2}+2 v_{n}\left\langle S p-p, y_{n}-p\right\rangle \\
& \leq\left(1-v_{n}\right)\left\|w_{n}-p\right\|^{2}+v_{n}\left\|S w_{n}-S p\right\|^{2}+2 v_{n}\left\langle S p-p, y_{n}-p\right\rangle \\
& \leq\left\|w_{n}-p\right\|^{2}+2 v_{n}\left\langle S p-p, y_{n}-p\right\rangle .
\end{aligned}
$$

Substituting (4.10) into (4.9), we have

$$
\begin{aligned}
\left\|x_{n+1}-p\right\|^{2} \leq & \left(1-\psi_{n}\right)\left\|w_{n}-p\right\|^{2}+\rho^{2} \psi_{n}\left\|x_{n}-p\right\|^{2} \\
& +2 \psi_{n}\left\langle f(p)-p, x_{n+1}-p\right\rangle+2\left(1-\psi_{n}\right) v_{n}\left\langle S p-p, y_{n}-p\right\rangle \\
\leq & \left(1-\left(1-\rho^{2}\right) \psi_{n}\right)\left\|x_{n}-p\right\|^{2}+2 \psi_{n}\left\langle f(p)-p, x_{n+1}-p\right\rangle \\
& +2\left(1-\psi_{n}\right) v_{n}\left\langle S p-p, y_{n}-p\right\rangle+2 \delta_{n}\left\langle x_{n}-x_{n-1}, w_{n}-p\right\rangle .
\end{aligned}
$$

From condition (C5) and the fact that $\left\{w_{n}\right\}$ is bounded, one has

$$
\limsup _{n \rightarrow \infty} 2 \delta_{n}\left\langle x_{n}-x_{n-1}, w_{n}-p\right\rangle=0 .
$$

By using Theorem 4.1, one observes that every weak cluster point of $\left\{x_{n}\right\}$ is in $\Omega$. Therefore, one easily infers from $p=\mathrm{P}_{\Omega} f(p)$ that

$$
\limsup _{n \rightarrow \infty}\left\langle f(p)-p, x_{n}-p\right\rangle \leq 0 .
$$

On the other hand, one observes that

$$
\left\langle S p-p, y_{n}-p\right\rangle=\left\langle S p-p, \mathrm{P}_{\mathrm{Fix}(T)} y_{n}-p\right\rangle+\left\langle S p-p, y_{n}-\mathrm{P}_{\mathrm{Fix}(T)} y_{n}\right\rangle .
$$

With the aid of $\mathrm{P}_{\mathrm{Fix}(T)} y_{n} \in \operatorname{Fix}(T)$, one has $\left\langle S p-p, \mathrm{P}_{\mathrm{Fix}(T)} y_{n}-p\right\rangle \leq 0$. Hence

$$
\begin{aligned}
\left\langle S p-p, y_{n}-p\right\rangle & \leq\left\langle S p-p, y_{n}-\mathrm{P}_{\mathrm{Fix}(T)} y_{n}\right\rangle \\
& \leq\|S p-p\|\left\|y_{n}-\mathrm{P}_{\mathrm{Fix}(T)} y_{n}\right\| \\
& \leq \frac{1}{k}\|S p-p\|\left\|y_{n}-T y_{n}\right\|
\end{aligned}
$$

From (4.1), we have

$$
\frac{x_{n+1}-x_{n}}{v_{n}}=\frac{\psi_{n}}{v_{n}}\left(f\left(x_{n}\right)-x_{n}\right)+\frac{\left(1-\psi_{n}\right)}{v_{n}}\left(T y_{n}-x_{n}\right),
$$


which together with $\lim _{n \rightarrow \infty} \frac{\left\|x_{n+1}-x_{n}\right\|}{v_{n}}=0$ and $\lim _{n \rightarrow \infty} \frac{\psi_{n}}{v_{n}}=0$ implies that $\lim _{n \rightarrow \infty} \frac{\left\|T y_{n}-x_{n}\right\|}{v_{n}}=0$. Hence,

$$
\lim _{n \rightarrow \infty} \frac{v_{n}\left\|T y_{n}-x_{n}\right\|}{\psi_{n}}=\lim _{n \rightarrow \infty} \frac{\left\|T y_{n}-x_{n}\right\|}{v_{n}} \frac{v_{n}^{2}}{\psi_{n}}=0 .
$$

From $y_{n}-w_{n}=v_{n}\left(S w_{n}-w_{n}\right)$, one has

$$
\lim _{n \rightarrow \infty} \frac{v_{n}}{\psi_{n}}\left\|y_{n}-w_{n}\right\|=\lim _{n \rightarrow \infty} \frac{v_{n}^{2}}{\psi_{n}}\left\|S w_{n}-w_{n}\right\|=0 .
$$

Therefore,

$$
\begin{aligned}
\frac{v_{n}}{\psi_{n}}\left\|y_{n}-T y_{n}\right\| & \leq \frac{v_{n}}{\psi_{n}}\left(\left\|y_{n}-w_{n}\right\|+\left\|w_{n}-x_{n}\right\|+\left\|x_{n}-T y_{n}\right\|\right) \\
& =\frac{v_{n}}{\psi_{n}}\left\|y_{n}-w_{n}\right\|+\frac{v_{n} \delta_{n}\left\|x_{n}-x_{n-1}\right\|}{\psi_{n}}+\frac{v_{n}}{\psi_{n}}\left\|x_{n}-T y_{n}\right\| \rightarrow 0, \text { as } n \rightarrow \infty .
\end{aligned}
$$

This further yields that

$$
\limsup _{n \rightarrow \infty} \frac{v_{n}}{\psi_{n}}\left\langle S p-p, y_{n}-p\right\rangle \leq 0 .
$$

Finally, by (4.11), (4.12), (4.13), (4.14) and using Lemma 2.3, we conclude that $\left\{x_{n}\right\}$ converges to $p$ in norm. The proof is completed.

Remark 4.2. (i) Prototypes for the iterative parameters are, for instance, $v_{n}=\frac{1}{n^{a}}, \psi_{n}=\frac{1}{n^{b}}$ and $\xi_{n}=\frac{1}{n^{c}}$, where $a \in\left(0, \frac{1}{3}\right), b \in(a, 2 a)$ and $c \in(a+b, \infty)$ or $a \in\left(\frac{1}{3}, \frac{1}{2}\right), b \in(a, 1-a)$ and $c \in(a+b, \infty)$.

(ii) If $\delta_{n}=0$ in (4.1), then Algorithm ITWIA (4.1) becomes the Algorithm TWIA (1.3) proposed by Yao, Liou and Marino [26].

\section{NUMERICAL EXPERIMENTS}

In this section, we provide several numerical experiments to demonstrate the computational performance of the proposed algorithms IVIA (3.1), ITWIA (4.1), and compare them with some existing algorithms, including Algorithm VIA (1.2) and Algorithm TWIA (1.3). All the programs are performed in MATLAB2018a on a PC Desktop Intel(R) Core(TM) i5-8250U CPU @ $1.60 \mathrm{GHz} 1.800 \mathrm{GHz}$, RAM 8.00 GB. MATLAB codes to reproduce the experiments are freely available at https://github.com/bingtan72/Tan2020iManns4HFPPs.

Let $C$ be a nonempty convex closed set in a real Hilbert space $H$. We consider the variational inequality problem (in short, VIP):

$$
\text { find } x^{*} \in C \quad \text { such that }\left\langle A\left(x^{*}\right), x-x^{*}\right\rangle \geq 0, \quad \forall x \in C,
$$

where $A: H \rightarrow H$ is a mapping. Denote by $\operatorname{VI}(C, A)$ the solution set of (VIP). Define $T: H \rightarrow H$ by $T:=P_{C}$ and $S: H \rightarrow H$ by $S:=I-\gamma A$, where $0<\gamma<2 / L$ ( $L$ is the Lipschitz constant of mapping $A)$. We see that Fix $\left(P_{C}(I-\gamma A)\right)=\mathrm{VI}(C, A)$. Therefore, variational inequality problem (VIP) is a special case of hierarchical fixed point problem (HFPP).

Example 5.1. Take $A: R^{2} \rightarrow R^{2}$ as follows:

$$
A(x, y)=(2 x+2 y+\sin (x),-2 x+2 y+\sin (y)), \quad \forall x, y \in R .
$$


Let $C=\left\{x \in R^{2} \mid-10 \mathbf{e} \leq x \leq 10 \mathbf{e}\right\}$, where $\mathbf{e}=(1,1)^{\top}$. It is not hard to check that $A$ is Lipschitz continuous with constant $L=\sqrt{26}$ and 1-strongly monotone [8]. Therefore, (VIP) has a unique solution $x^{*}=(0,0)^{\top}$.

Our parameter are set as follows. In all algorithms, set $v_{n}=(n+1)^{-1 / 6}, \psi_{n}=(n+1)^{-1 / 4}$, $f(x)=0.5 x$ and $\gamma=0.9 / L$. In Algorithm IVIA (3.1) and Algorithm ITWIA (4.1), we update the inertial parameter $\delta_{n}$ through (3.6), where $\xi_{n}=(n+1)^{-1 / 2}$ and $\delta=0.5$. Let $x_{-1}=x_{0}$ be randomly generated by the MATLAB function $k \times \operatorname{rand}(m, 1)$ (where Case I: $k=10$, Case II: $k=100$, Case III: $k=1000$, and Case IV: $k=10000)$. Denote by $E_{n}=\left\|x_{n}-x^{*}\right\|_{2}$ the error of the iterative algorithms and $E_{n}<10^{-8}$ the common stopping criterion. Our numerical results are shown in Table 1 and Fig. 1.

TABLE 1. Compare the number of iterations for Example 5.1

\begin{tabular}{ccccc}
\hline Cases & ITWIA (4.1) & IVIA (3.1) & TWIA (1.3) & VIA (1.2) \\
\hline I & 28 & 28 & 42 & 42 \\
II & 33 & 34 & 46 & 47 \\
III & 36 & 39 & 49 & 51 \\
IV & 39 & 43 & 52 & 56 \\
\hline
\end{tabular}

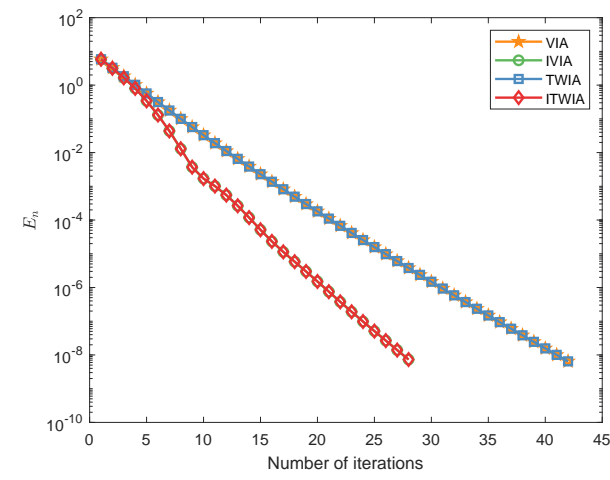

(a) Case I

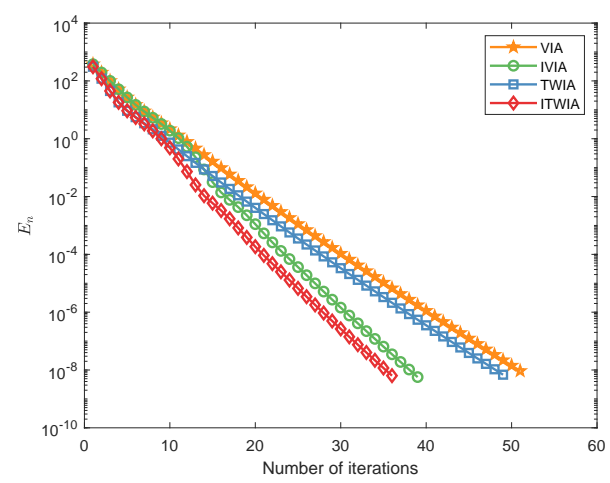

(c) Case III

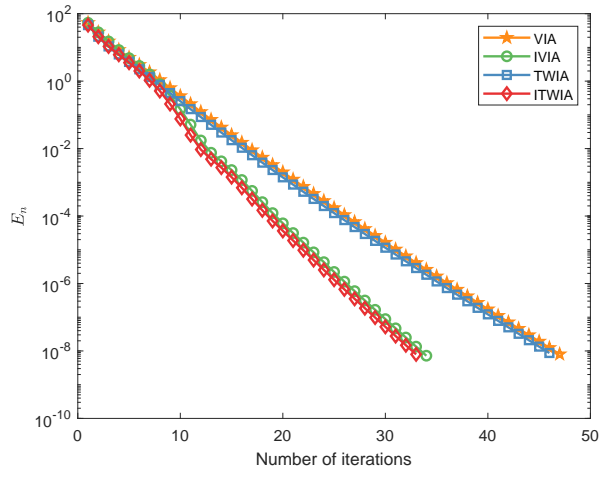

(b) Case II

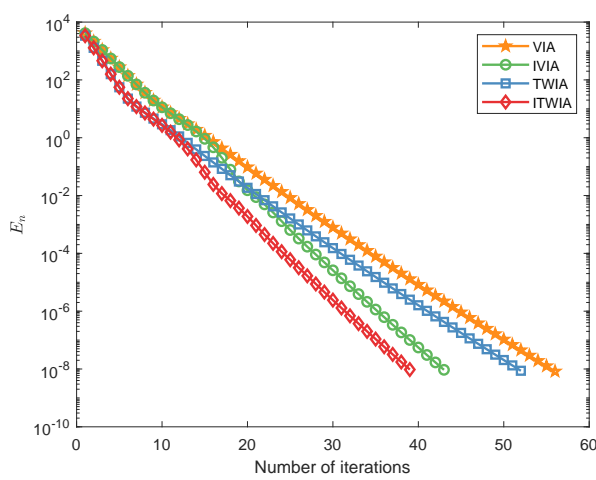

(d) Case IV

FIGURE 1. Numerical behavior of $\left\{E_{n}\right\}$ with different initial values for Example 5.1 
Further, we use the control variable method for Algorithm IVIA (3.1) and Algorithm ITWIA (4.1) to analyze the variables $v_{n}, \psi_{n}, \xi_{n}, \delta, f(x)$ and $\gamma$, respectively. Fig. 2(a), Fig. 2(b), Fig. 2(c), Fig. 2(d), Fig. 3 and Fig. 4 show the effect of different $v_{n}, \psi_{n}, \xi_{n}, \delta, f(x)$ and $\gamma$ on the iteration error $E_{n}$ when other parameters remain the same as in Example 5.1.

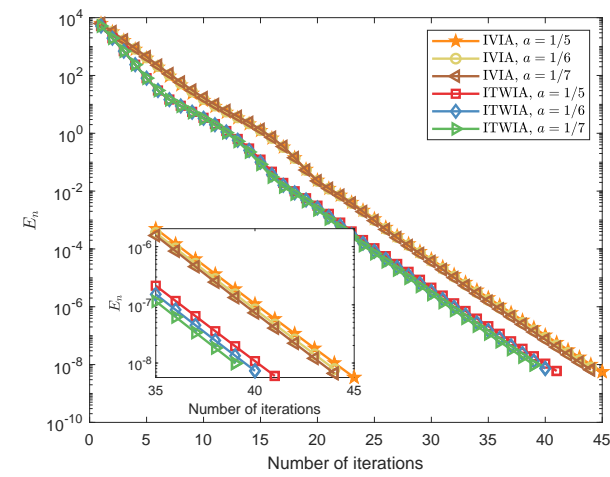

(a) Different $v_{n}=\frac{1}{(n+1)^{a}}$

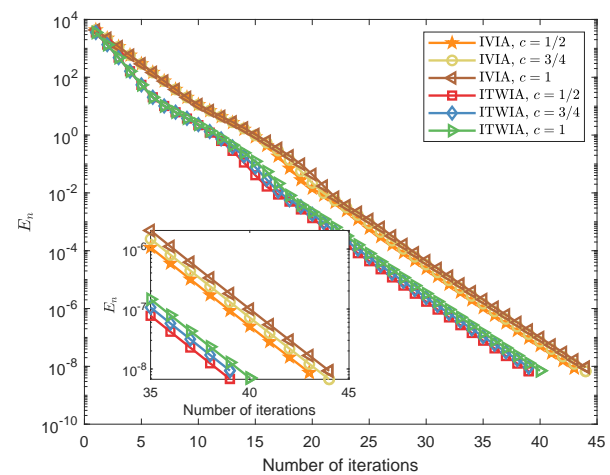

(c) Different $\xi_{n}=\frac{1}{(n+1)^{c}}$

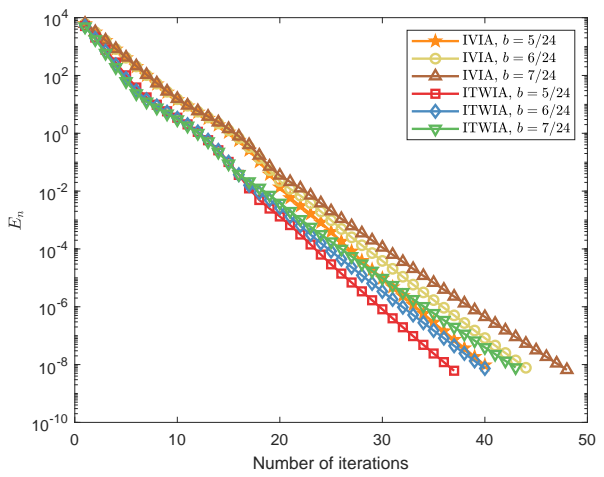

(b) Different $\psi_{n}=\frac{1}{(n+1)^{b}}$

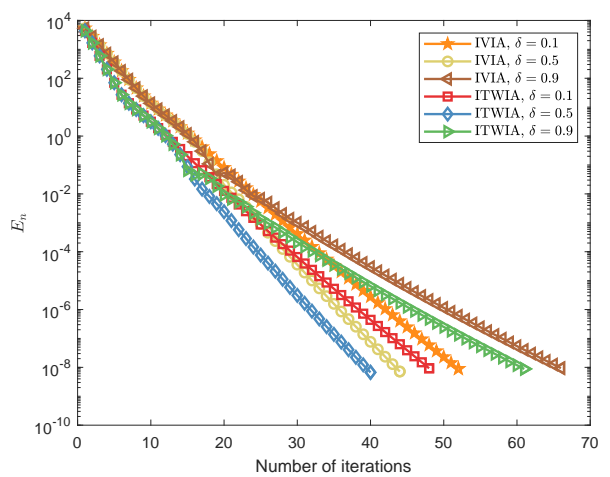

(d) Different $\delta$ in (3.6)

FIGURE 2. Numerical behavior of $\left\{E_{n}\right\}$ with different parameters for Example 5.1

Example 5.2. We consider the linear operator $A(x)=M x+q$, where $q \in R^{m}$ and

$$
M=N N^{\top}+U+D,
$$

where $N$ is a $m \times m$ matrix, $U$ is a $m \times m$ skew-symmetric matrix, and $D$ is a $m \times m$ diagonal matrix with its diagonal entries being nonnegative (hence $M$ is positive symmetric definite). The feasible set $C$ is given by $C=\left\{x \in R^{m}:-5 \leq x_{i} \leq 5, i=1, \ldots, m\right\}$. It is clear that $A$ is Lipschitz monotone with constant $L=\|M\|$. In the experiment, $D$ is generated randomly in $[1,5]$, and all entries of $N, U$ are generated randomly and uniformly in $[-5,5]$. Let $q=0$. Then the solution set is $x^{*}=\{\boldsymbol{0}\}$. Our parameter are set as follows. In all algorithms, set $v_{n}=(n+1)^{-1 / 6}$, $\psi_{n}=(n+1)^{-1 / 4}, f(x)=0.5 x$ and $\gamma=0.9 / L$. In Algorithm IVIA (3.1) and Algorithm ITWIA (4.1), we update the inertia parameter $\delta_{n}$ through (3.6), where $\xi_{n}=(n+1)^{-1 / 2}$ and $\delta=0.5$. Let $x_{-1}=x_{0}$ be randomly generated by $10^{4} \times \operatorname{rand}(m, 1)$. We test the convergence behavior under different dimensions parameters $m$. Numerical results are reported in Table 2 and Fig. 5. 


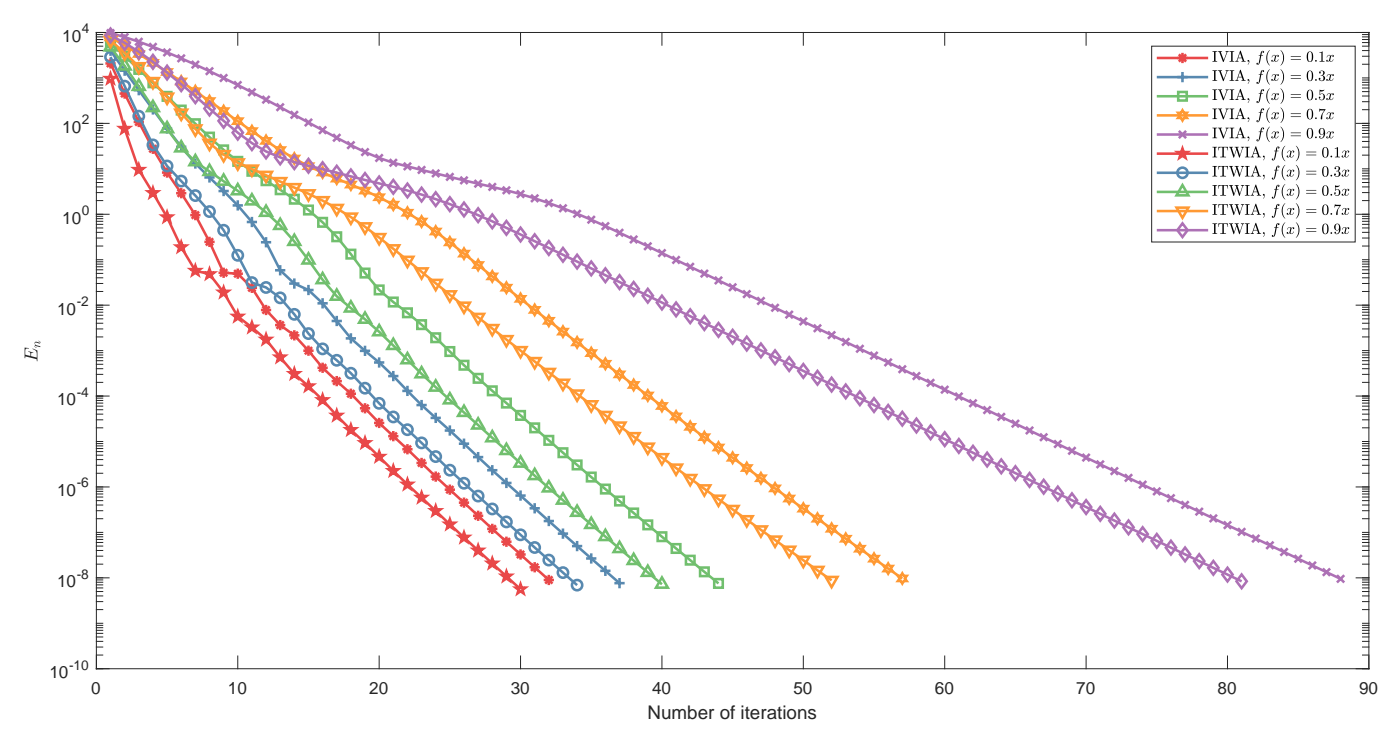

FIGURE 3. Numerical behavior of $\left\{E_{n}\right\}$ with different $f(x)$ for Example 5.1

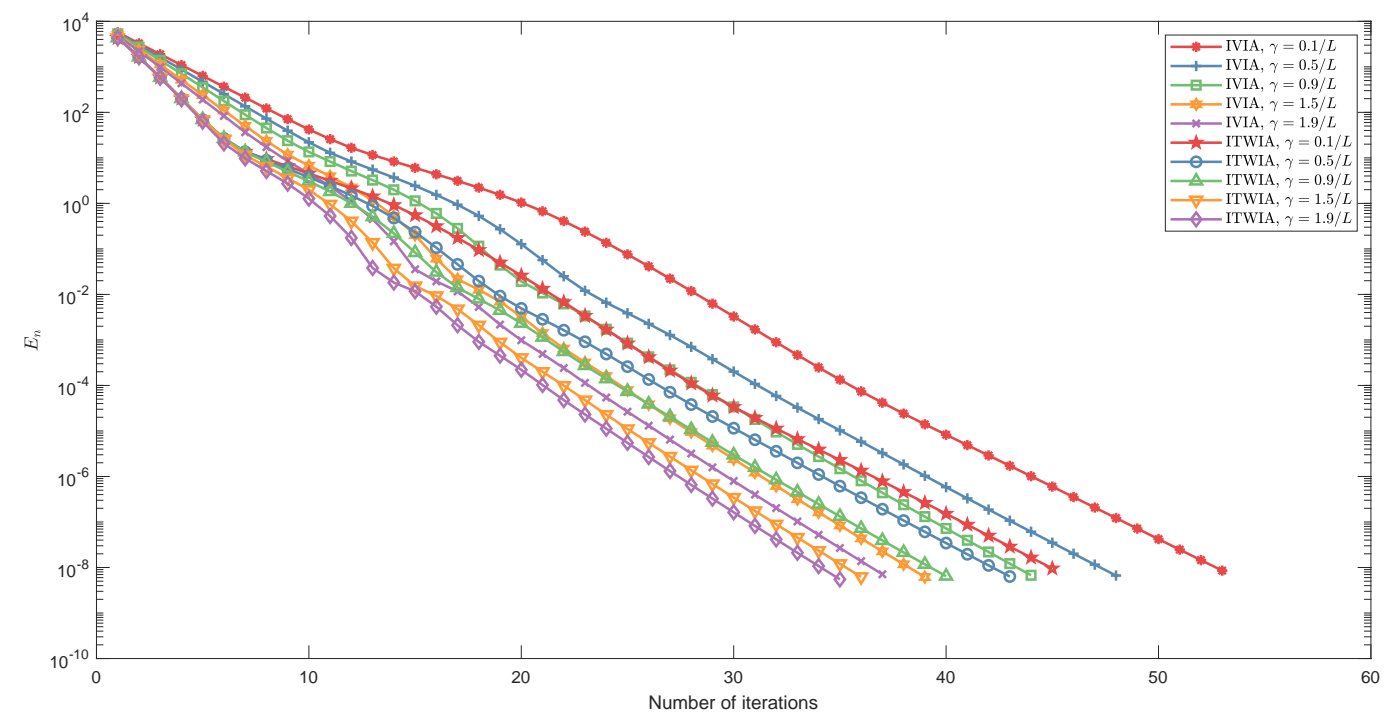

FIGURE 4. Numerical behavior of $\left\{E_{n}\right\}$ with different stepsize $\gamma$ for Example 5.1

TABLE 2. Compare the number of iterations for Example 5.2

\begin{tabular}{ccccc}
\hline Dimensional & ITWIA (4.1) & IVIA (3.1) & TWIA (1.3) & VIA (1.2) \\
\hline 20 & 44 & 54 & 97 & 110 \\
100 & 50 & 60 & 105 & 118 \\
500 & 54 & 65 & 109 & 123 \\
1000 & 56 & 67 & 112 & 126 \\
\hline
\end{tabular}




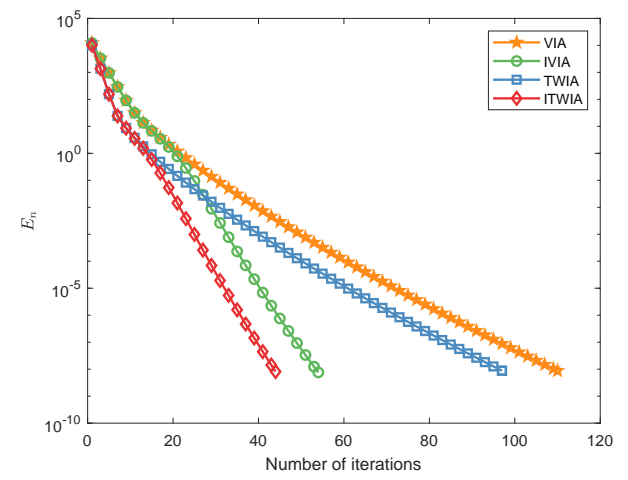

(a) Dimensional parameters $m=20$

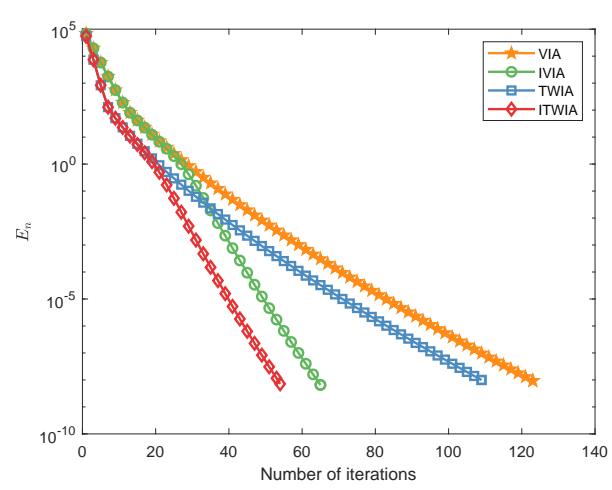

(c) Dimensional parameters $m=500$

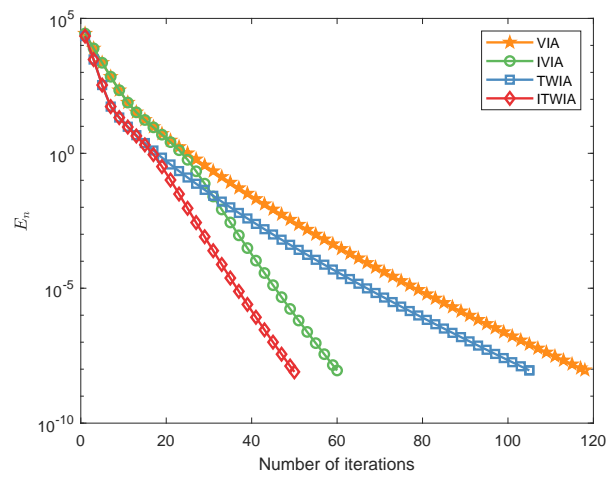

(b) Dimensional parameters $m=100$

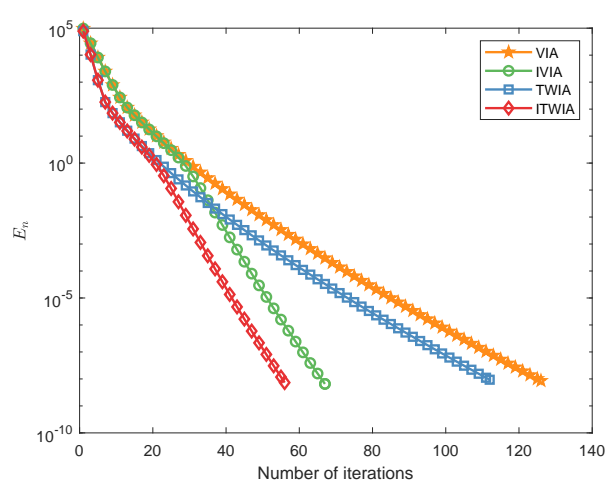

(d) Dimensional parameters $m=1000$

FIGURE 5. Numerical behavior of $\left\{E_{n}\right\}$ with different dimensions $m$ for Example 5.2

Example 5.3. Suppose that $H=L^{2}([0,1])$ with inner product $\langle x, y\rangle:=\int_{0}^{1} x(t) y(t) \mathrm{d} t, x, y \in H$ and norm $\|x\|:=\left(\int_{0}^{1}|x(t)|^{2} \mathrm{~d} t\right)^{1 / 2}$. Let $C:=\{x \in H:\|x\| \leq 1\}$ be the unit ball. Define an operator $A: C \rightarrow H$ by

$$
A(x)(t)=\int_{0}^{1}(x(t)-G(t, v) g(x(v))) \mathrm{d} v+h(t), \quad t \in[0,1], x \in C,
$$

where

$$
G(t, v)=\frac{2 t v \mathrm{e}^{t+v}}{e \sqrt{\mathrm{e}^{2}-1}}, \quad g(x)=\cos x, \quad h(t)=\frac{2 t \mathrm{e}^{t}}{e \sqrt{\mathrm{e}^{2}-1}} .
$$

It is known that $A$ is monotone and $L$-Lipschitz continuous with $L=2$ and $x^{*}=\{\boldsymbol{0}\}$ is the solution of the corresponding variational inequality problem. Numerical results are given in Table 3 and Fig. 6. In Table 3, "Iter." and "Times(s)" denote the number of iterations and the cpu time in seconds, respectively.

Remark 5.1. (1) We observe from numerical results of Examples 5.1-5.3 that our proposed Algorithm IVIA (3.1) and Algorithm ITWIA (4.1) are efficient and very fast. In addition, our algorithms converge faster than Algorithm VIA (1.2) and Algorithm TWIA (1.3) .

(2) Our proposed algorithms are strong robust, and initial values and scale of the dimension does not affect the performance of our algorithms. 
TABLE 3. Compare different algorithms with different initial values for Example 5.3

\begin{tabular}{|c|c|c|c|c|c|c|c|c|c|}
\hline \multirow{2}{*}{ Cases } & \multirow{2}{*}{ Initial values } & \multicolumn{2}{|c|}{ ITWIA (4.1) } & \multicolumn{2}{|c|}{ IVIA (3.1) } & \multicolumn{2}{|c|}{ TWIA (1.3) } & \multicolumn{2}{|c|}{ VIA (1.2) } \\
\hline & & Iter. & Time(s) & Iter. & Time(s) & Iter. & Time(s) & Iter. & Time(s) \\
\hline I & $\begin{array}{l}x_{-1}=10 \cos (3 \pi t) \\
x_{0}=10 \sin (3 \pi t)\end{array}$ & 10 & 3.7971 & 10 & 3.7056 & 20 & 6.6083 & 21 & 7.6692 \\
\hline II & $\begin{array}{l}x_{-1}=10 t^{2} \\
x_{0}=50 e^{t}\end{array}$ & 16 & 5.7189 & 17 & 6.3725 & 23 & 8.9447 & 25 & 10.1334 \\
\hline III & $\begin{array}{l}x_{-1}=100 t^{2} \\
x_{0}=100 e^{t}+t^{4} / 24\end{array}$ & 16 & 5.5771 & 18 & 5.9763 & 24 & 8.3232 & 26 & 9.3359 \\
\hline IV & $\begin{array}{l}x_{-1}=1000 e^{t} \\
x_{0}=1000 e^{t}+t^{4} / 24\end{array}$ & 18 & 6.2018 & 21 & 6.8170 & 26 & 8.9705 & 29 & 10.3813 \\
\hline
\end{tabular}

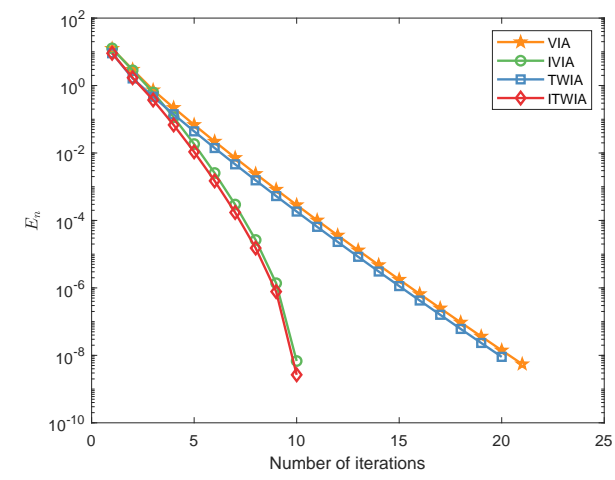

(a) Case I

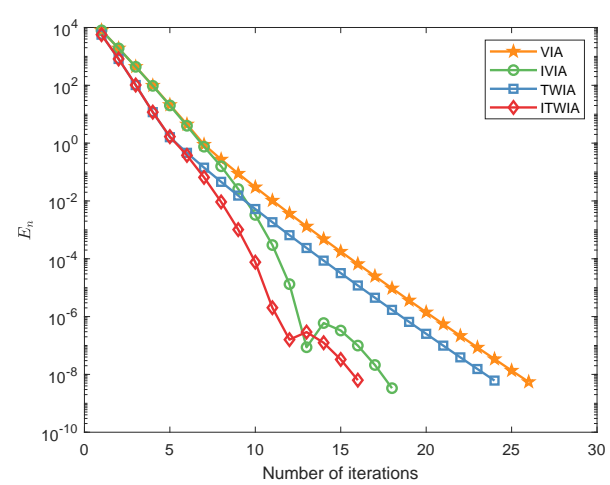

(c) Case III

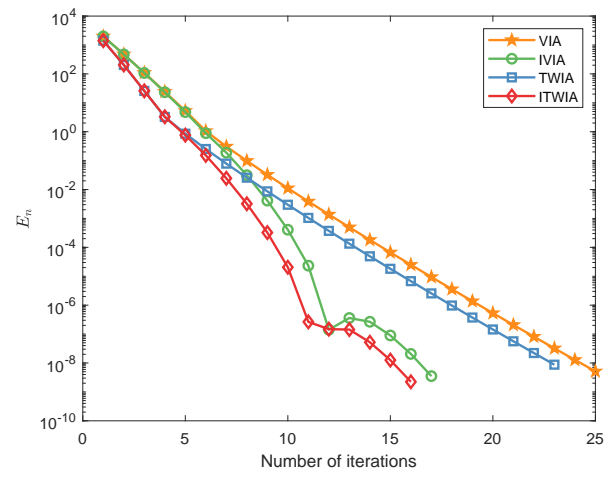

(b) Case II

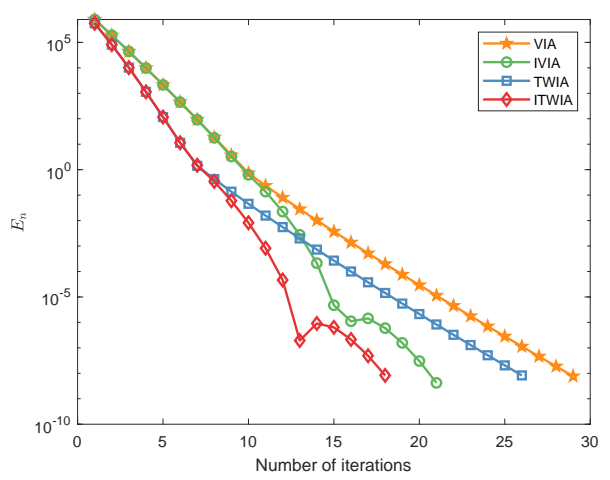

(d) Case IV

FIGURE 6. Numerical behavior of $\left\{E_{n}\right\}$ with different initial points for Example 5.3

(3) It should be noted that our initial values selection are very large (see Fig. 1, Fig. 5 and Fig. 6), and our dimensions are also chosen very large (see Table 2). Even so, our algorithms can still achieve accuracy requirements with a small number of iterations.

(4) As show in Fig. 6, in a real Hilbert space, our algorithms converge very quickly and are independent of the initial values. 


\section{THE CONCLUSION}

In this paper, we investigated the hierarchical fixed point problems. We modified the Algorithm VIA (1.2) and the Algorithm TWIA (1.3) with the aid of the inertial idea. Strong convergence was established under some mild assumptions in real Hilbert spaces. Numerical experiments show that our algorithms, which improve and generalize some of the existing algorithms, are very efficient and robust.

\section{REFERENCES}

[1] H. Attouch, H. Riahi, M. Théra, Somme ponctuelle d'opérateurs maximaux monotones, Serdica Math. J. 22 (1996), 267-292.

[2] H.H. Bauschke, P.L. Combettes, Convex Analysis and Monotone Operator Theory in Hilbert Spaces, Springer, New York, 2011.

[3] A. Beck, M. Teboulle, A fast iterative shrinkage-thresholding algorithm for linear inverse problems, SIAM J. Imaging Sci. 2 (2009), 183-202.

[4] H. Brezis, Opérateurs Maximaux Monotones et Semi-groupes de Contractions dans les Espaces de Hilbert, Elsevier, 1973.

[5] S.Y. Cho, X. Qin, L. Wang, Strong convergence of a splitting algorithm for treating monotone operators, Fixed Point Theory Appl. 2014 (2014), Article ID 94.

[6] S.Y. Cho, A convergence theorem for generalized mixed equilibrium problems and multivalued asymptotically nonexpansive mappings, J. Nonlinear Convex Anal. 21 (2020), 1017-1026.

[7] Q.-L. Dong, K.R. Kazmi, R. Ali, X.H. Li, Inertial Krasnosel'skiı̌-Mann type hybrid algorithms for solving hierarchical fixed point problems, J. Fixed Point Theory Appl. 21 (2019), Article ID 57.

[8] Q.-L. Dong, Y.J. Cho, L.L. Zhong, T.M. Rassias, Inertial projection and contraction algorithms for variational inequalities, J. Global Optim. 70 (2018), 687-704.

[9] J. Fan, L. Liu, X. Qin, A subgradient extragradient algorithm with inertial effects for solving strongly pseudomonotone variational inequalities, Optimization 69 (2019), 2199-2215.

[10] L. Liu, X. Qin, On the strong convergence of a projection-based algorithm in Hilbert spaces, J. Appl. Anal. Comput. 10 (2019), 104-117.

[11] A. Moudafi, P.E. Maingé, Towards viscosity approximations of hierarchical fixed-point problems, Fixed Point Theory Appl. 2006 (2006), Article ID 95453.

[12] A. Moudafi, Krasnoselski-Mann iteration for hierarchical fixed-point problems, Inverse Probl. 23 (2007), 1635-1640.

[13] P.E. Maingé, A. Moudafi, Strong convergence of an iterative method for hierarchical fixed-point problems, Pac. J. Optim. 3 (2007), 529-538.

[14] B.T. Polyak, Some methods of speeding up the convergence of iteration methods, USSR Comput. Math. Math. Phys. 4 (1964), 1-17.

[15] X. Qin, S.Y. Cho, L. Wang, A regularization method for treating zero points of the sum of two monotone operators, Fixed Point Theory Appl. 2014 (2014), Article ID 75.

[16] X. Qin, S.Y. Cho, L. Wang, Strong convergence of an iterative algorithm involving nonlinear mappings of nonexpansive and accretive type, Optimization 67 (2018), 1377-1388.

[17] X. Qin, L. Wang, J.C. Yao, Inertial splitting method for maximal monotone mappings, J. Nonlinear Convex Anal. 21 (2020), 2325-2333.

[18] D.R. Sahu, J.C. Yao, M. Verma, K.K. Shukla, Convergence rate analysis of proximal gradient methods with applications to composite minimization problems, Optimization (2020), doi:10.1080/02331934.2019.1702040.

[19] B. Tan, Z. Zhou, S. Li, Strong convergence of modified inertial Mann algorithms for nonexpansive mappings, Mathematics 8 (2020), Article ID 462.

[20] B. Tan, S. Xu, S. Li, Inertial shrinking projection algorithms for solving hierarchical variational inequality problems, J Nonlinear Convex Anal. 21 (2020), 871-884.

[21] B. Tan, S. Xu, Strong convergence of two inertial projection algorithms in Hilbert spaces, J. Appl. Numer. Optim. 2 (2020), 171-186. 
[22] W. Takahahsi, J.C. Yao, The split common fixed point problem for two finite families of nonlinear mappings in Hilbert spaces, J. Nonlinear Convex Anal. 20 (2019), 173-195.

[23] W. Takahashi, C.F. Wen, J.C. Yao, The shrinking projection method for a finite family of demimetric mappings with variational inequality problems in a Hilbert space, Fixed Point Theory 19 (2018), 407-419.

[24] H.K. Xu, Iterative algorithms for nonlinear operators, J. London Math. Soc. 66 (2002), 240-256.

[25] Y. Yao, Y.C. Liou, Weak and strong convergence of krasnoselski-Mann iteration for hierarchical fixed point problems, Inverse Probl. 24 (2008), 015015 (8pp).

[26] Y. Yao, Y.C. Liou, G. Marino, Two-step iterative algorithms for hierarchical fixed point problems and variational inequality problems, J. Appl. Math. Comput. 31 (2009), 433-445.

[27] Z. Zhou, B. Tan, S. Li, A new accelerated self-adaptive stepsize algorithm with excellent stability for split common fixed point problems, Comput. Appl. Math. 39 (2020), Article ID 220. 